\title{
Aprendizagem aplicada à análise da síndrome de Burnout em Técnicos
}

\section{da Atenção Primária à Saúde}

El aprendizaje aplicado al análisis del síndrome de Burnout en Técnicos de Atención

Primaria de Salud

Learning applied to the analysis of Burnout syndrome in Primary Health Care Technicians.

\section{Luis Antonio Álvarez-Silva}

Master. Instituto Superior Tecnológico Vicente Rocafuerte, lalvarez@itsvr.edu.ec, Guayaquil - Ecuador, https://orcid.org/0000-0001-5835-714X

\footnotetext{
Recibido 8 Março de 2019 - Aceito em 21 de abril de 2020

Formación docente - revista iberoamericana de educación

http://www.revista-iberoamericana.org/index.php/es/index https://creativecommons.org/licenses/by/4.0/deed.es e-ISSN: 2737-632X
}

Vol - 3 No. 2, abril - junho de 2020

Pags 51 - 68

Resumo. Atualmente, os computadores são usados não apenas como equipamento de suporte para diferentes tarefas humanas, mas como um meio de obter informações, por sua vez, a computação está presente em quase todos os campos da vida moderna. Este artigo trata de crimes de computador que conseguem ser considerados crimes eletrônicos, tão graves que se tornam um problema genérico para o avanço da ciência da computação. Esses crimes têm consigo o que é fraude, roubo, falsificação (documentos), etc. $\mathrm{O}$ exemplo mais claro é quando uma pessoa é um homem ou uma mulher que rouba informações e causa danos a servidores ou computadores que se tornam a maioria virtual da informação em formato digital, cujo dano se torna cada vez maior. Podemos apreciar a análise das ações realizadas por vários países para regular essa incidência nas redes digitais e, por sua vez, dar recomendações para que os usuários adotem para melhorar sua experiência enquanto navegam nessa rede. Na pesquisa realizada, temos como pontos-chave a 
realização de uma investigação bibliográfica da arte do crime de computador no Equador, que a comparou com a legislação de outros países, na qual se verá que ainda temos muito a mudar. Nas conclusões apresentadas, devemos reestruturar seu Código Penal Orgânico Abrangente (COIP) e ter mais severidade para quem infringe a lei o roubo de informações, o desvio de dinheiro, a quem é confiado o dinheiro dos usuários, dos quais é uma questão de estudo, que não apenas o representante legal seja investigado e sancionado, mas também o funcionário bancário que fornece ou acessa as informações do banco de dados das diferentes instituições financeiras do Equador.

Palavras-chave: atenção Primária, Saúde, Síndrome de Burnout, Trabalho, Técnicos.

Resumen Actualmente las computadoras se utilizan no sólo como equipos de soporte a diferentes diligencias humanas, sino como medio obtener información, a su vez la informática está presente en casi todos los campos de la vida moderna. El presente artículo aborda los delitos informáticos que logran ser considerados como crímenes electrónicos, tan graves que consiguen llegar a ser un genérico problema para el avance de la informática. Estos delitos tienen consigo lo que es fraude, robo, falsificación (documentos), etc. El ejemplo más claro es cuando una persona sea hombre o mujer consigue robar información y causar daños de servidores o computadores que llegan ser la mayoría virtuales para la información está de forma digital cuyo daño cada vez se vuelve más grande. Podemos apreciar, el análisis de las acciones realizadas por parte de diversos países para regular esta incidencia en las redes digitales y a su vez dar recomendaciones para que los usuarios puedan adoptar para mejorar su experiencia mientras navegan en esta red. En la investigación realizada tenemos como puntos clave el realizar una investigación bibliográfica del arte de los delitos informáticos en el 
Ecuador, quienes lo hemos comparado con la legislación de otros países en la cual se verá que aún tenemos mucho que cambiar. En las conclusiones dadas están que nuestro debería de reestructurar su Código Orgánico Integral Penal(COIP), y el tener más severas para quienes infrinjan las ley el hurto de información, el desvío de dinero, a quienes se les confía el dinero de los usuario, de lo cual es materia de estudio, que no solo sea investigado y sancionado el representante legal si no también el funcionario bancario que dé o accese a la información de la base de datos de las diferentes instituciones financieras en el Ecuador.

Palabras clave: atención Primaria, Salud, Síndrome de Burnout, Trabajo, Técnicos.

Abstract The work situation of the organizations causes stress in their collaborators, in some cases this improves their skills and in another case causes wear. The objective of this article is to identify the level of Burnout Syndrome (attrition) that the Technicians in Primary Health Care have; who are a new typology of professionals from Ecuador, in what concerns the last years. The study is of a non-experimental cross-sectional nature, in which the sample consisted of 187 TAPS that fulfill the mission of managing and promoting health through multi-faceted processes in their respective communities. For this, one of the most used tools was used for the analysis of this phenomenon, which is the Maslach Burnout Inventory (MBI). The application of this tool gave a reliability score of 0.89 according to Cronbach's Alpha. In conclusion, the subject of Burnout continues being something unknown for the organizations; In addition, this syndrome is already present in the TAPS at a mid-range level; therefore, it can move forward if necessary steps are not taken.

Key words: Primary Care, Health, Burnout Syndrome, Work, Technicians 


\section{INTRODUÇÃO}

Há mais de 72 anos, na Conferência Sanitária Internacional realizada em Nova York, a Organização Mundial da Saúde (OMS) definiu a palavra Saúde oficial, que transcendeu diferentes países, culturas, políticas, etc. Essa definição não apenas a engloba como resultado biológico, mas a representa como a integração de três elementos inseparáveis; São eles: físico (biológico), mental (psicológico) e social (Organização Mundial da Saúde, 2018).

Graças a essa definição, muitos dos países pertencentes às Nações Unidas adotaram mudanças em suas políticas públicas; É o caso do Equador, que defende a perspectiva da saúde há mais de 60 anos, e não apenas a ausência de doenças ou condições. Por esse motivo, o artigo 32 da Constituição equatoriana indica que o bem-estar saudável é um direito fundamental no qual o Estado exerce uma função de garantia para o povo equatoriano e que a eficácia deste artigo está inextricavelmente relacionada ao bom viver (Constituição da República do Equador, 2008).

Desde 2012, o Ministério da Saúde Pública do Equador apresentou o Modelo de Atenção Integral à Saúde (MAIS), alinhado aos objetivos do milênio de exercer uma relação direta com a noção de perspectiva de desenvolvimento humano sustentável. Para isso, este modelo propõe que, para o melhor cumprimento do artigo 32 da Constituição do Equador, o bem-estar saudável deve ser orientado para a fundação, diligência e compensação do bem-estar saudável em todas as suas manifestações (biológicas, psicológicas e sociais); tomando como foco de atenção o indivíduo como tal, a família e a comunidade do ponto de vista organizacional, ou seja, considerando todas as 
modalidades de serviços integrais, intersetorialidade e participação da própria comunidade (Ministério da Saúde Pública - MAIS, 2012).

Sob essa abordagem de saúde pública, o Estado equatoriano aprovou novos processos para a formação de profissionais no Sistema Público de Saúde, cujo nome é TAPS, cujas iniciais significam Técnico de Atenção Básica, que faria parte das equipes de atenção integral; tomando como referência os modelos de países como Brasil, Costa Rica e Cuba, que administram o esquema.

A formação dos profissionais do TAPS possui as características de um ensino superior, que possui um rigoroso processo de treinamento e prática. No momento de seu processo de conclusão, o TAPS possui as competências técnicas e comportamentais para enfrentar a gestão abrangente da assistência proposta pelo MAIS.

Atualmente, existem mais de 2000 TAPS registrados no sistema de ensino superior equatoriano, que diariamente cumprem um manual de funções ajustado à gestão da saúde pública. Nesta gestão, o TAPS é apresentado diariamente para diferentes situações de atenção aos usuários, seja no campo ou no campo.

Com efeito, a atividade que realizam não está isenta de situações de riscos ocupacionais, como a Síndrome de Burnout ou também chamada de síndrome da fadiga do trabalho, que ocorre repetidamente em trabalhadores expostos ao serviço de atenção à saúde. usuários (Patlán, 2013). Além disso, é um fenômeno que ocorre especialmente em pessoas que prestam serviços abrangentes, uma vez que é um grupo vulnerável testemunhar esse tipo de evento devido a seus esforços tradicionais (Domínguez-González, VelascoJiménez, Meneses-Ruiz, Valdivia-Gómez, \& Castro-Martínez, 2017).

Essa síndrome pode ser definida como um distúrbio psicológico que envolve fatores de fadiga emocional, sentimentos de desamparo e perda de ideais; tudo 
isso como resultado da sobrecarga de trabalho e da exposição constante ao estresse no trabalho (Albendín, Gómez, Cañadas, San Luis e Aguayo, 2016). Além disso, hoje é importante prestar atenção ao tema organizacional dos processos de trabalho em que o estresse no trabalho e outros riscos psicossociais estão incorporados, que a longo prazo estão deteriorando o bemestar dos trabalhadores, ainda mais para aqueles que estão expostos a situações. atenção ao usuário, como é o caso dos profissionais de saúde; Por esse motivo, Burnout se torna um problema de atenção urgente (MunaycoGuillén, Cámara-Reyes e Torres-Romucho, 2014).

No entanto, a definição mais aceita de Síndrome de Burnout é a dos autores Maslach e Jackson, na qual apontam que a Síndrome de Burnout é o estado de exaustão emocional, desânimo, desmotivação, sentimento de falta de realização pessoal e atitude pessimista. do sujeito antes da relação de emprego (Gómez-Urquiza, et al., 2017).

Este artigo, por si só, visa identificar, através de um processo de investigação científica, o nível de Burnout que os Técnicos da Atenção Básica possuem, por serem profissionais de Saúde, expostos às condições de trabalho exigidas; quais muitos estão no campo, na comunidade e em muitas ocasiões no nível administrativo; em que os principais beneficiários são os usuários das diferentes unidades operacionais e subcentros das diferentes localidades. A estrutura conceitual do Modelo de Atenção Integral à Família, Comunidade e Saúde Intercultural baseia-se nos artigos $n^{\circ} 32,35,66,361$, nos quais o Estado equatoriano enfatiza: garantir o direito ao bem-estar saudável, serviço especializado a grupos de atenção prioritária (Constituição da República do Equador, 2008). Além disso, o plano nacional de Bem Viver, em seus objetivos $n^{\circ} \mathrm{s}$ 1, 2, 3 e 4, enfatiza que o Estado equatoriano garante o direito a um Bom Viver, sendo este um ponto-chave para alcançar a visão do país (Ministério da Saúde Pública - MAIS, 2012). 
A lei de saúde orgânica, em seus artigos $n^{\circ} \mathrm{s} 6,10,69$, indica que o Ministério da Saúde do Equador é responsável por: treinar e executar programas de assistência integral a todos os indivíduos em todas as etapas de sua vida. ; implementar políticas estaduais de saneamento e controle de doenças. Os Objetivos do Milênio propostos pelos países membros das Nações Unidas, nos quais os pontos mais relacionados ao bem-estar integral se concentram nos objetivos $n^{\circ}$ s 4, 5 e 6, que apontam para a redução de óbitos em crianças menores de 5 anos, melhorar o bem-estar saudável das mães nos estágios gestacionais e pós-gestacionais, minimizar a disseminação da malária, HIV, malária, entre outros (Organização Pan-Americana da Saúde - OPAS, 2008). Consequentemente, o Modelo de Atenção Integral, Familiar, Comunitária e Intercultural tem quatro fatores principais como determinantes: os aspectos comportamentais, biológicos, ambientais e sociais. O fator comportamental refere-se aos hábitos e comportamentos dos sujeitos em relação à sua relação com a Saúde; o fator biológico refere-se aos aspectos genéticos, nutricionais, idade e gênero; o fator ambiental refere-se aos elementos da natureza que influenciam o bem-estar dos sujeitos; e o fator social refere-se às condições externas que influenciam os estados de saúde, como situação financeira, trabalho, valores (Ministério da Saúde Pública - MAIS, 2012).

A abordagem do modelo, por si só, enfatiza a gestão da Atenção Primária à Saúde Renovada, ou seja, alinhada à Promoção do bem-estar saudável como um processo de educação dos indivíduos sobre seus cuidados e os de sua família, a fim de prevenir afetações que podem causar efeitos negativos em seu estado físico, mental e social.

É então que esse programa fortalece e potencializa a promoção da saúde através de seus cuidados primários; É por isso que aparece o Técnico de Atenção Primária à Saúde, que contribui com as equipes de Médicos, Médicos Rurais, Médicos de Família, Médicos Comunitários, Enfermeiras, 
Psicólogos, Obstetras e outros profissionais, para a respectiva promoção, prevenção de doenças, altos riscos potenciais; envolvendo a comunidade, com o objetivo comum de melhorar suas condições.

Dentro das funções do TAPS, algumas atividades são incorporadas que levam a processos e ações em seu local de trabalho. A missão do Técnico de Atenção Básica em uma unidade ou sub-centro visa ser um aliado na identificação e intervenção de riscos nos níveis individual, familiar, comunitário e coletivo, enfatizando o Bom Viver; permanentemente usando os valores, princípios e abordagem cultural do atendimento integral a indivíduos em sua comunidade e população.

Para alcançar essa missão, é necessária a trajetória das atividades de campo ou de campo; para que o processo de identificação possa se materializar. Algumas dessas atividades são: análise de falhas no nível de saúde da comunidade, usando as ferramentas de estudo, levando em consideração um mapeamento da comunidade para alertar o foco da atenção para grupos vulneráveis; realizar a transferência adequada de informações para as unidades sobre o que foi identificado para uma organização adequada com a equipe multidisciplinar. Além disso, devem ser realizados projetos nos quais a comunidade intervenha para contribuir com seu estado de bem-estar integral; entre outras.

Em suma, o significado do TAPS reside em ser um elo entre o bem-estar saudável e a comunidade a ele atribuída; realizar a promoção correspondente, participar do diagnóstico, identificação, planejamento e execução das atividades locais do projeto; estabelecer canais de comunicação para fins benéficos entre a comunidade e a unidade correspondente; gerenciar o processo de treinamento e educação da comunidade sobre questões de saúde para conscientização e a respectiva mudança de atitudes que beneficiam o processo. 


\section{MATERIAIS E MÉTODOS}

Esse processo de pesquisa utilizado obedece a um delineamento transversal não experimental, uma vez que as informações foram coletadas em determinado momento, no qual não houve manipulação do fenômeno. Portanto, o escopo foi descritivo, uma vez que são especificadas as características da Síndrome de Burnout, o modelo de atenção integral e as funções do Técnico de Atenção Básica.

O processo de amostragem está alinhado à tipologia de amostragem não probabilística por conveniência, na qual os critérios de seleção dos pesquisadores são direcionados a determinadas características que a amostra deve possuir. Portanto, os critérios de seleção da amostra enfatizam que os membros que a compõem devem ter um tempo mínimo de um (1) ano em seu trabalho como TAPS e devem ser direcionados, principalmente, ao trabalho de campo (Hernández Sampieri, Fernández e Baptista, 2014).

Os critérios de seleção obtiveram que $95 \%$ deles atendem aos requisitos, o que corresponde a 187 técnicos. A característica é a seguinte: 32\% é masculino, enquanto $68 \%$ é feminino.

A idade da amostra está dividida da seguinte forma: 16\% têm entre 20 e 25 anos, $27 \%$ entre 26 e 30 anos, 25\% entre 31 e 35 anos, $18 \%$ entre 36 e 40 anos e 14\% têm mais de 41 anos de idade. A idade média da amostra é de 32 anos.

Em relação ao tempo de trabalho, a amostra apresenta as seguintes características: $26 \%$ possuem tempo de trabalho de 1 a 2 anos, $49 \%$ de 2 a 3 anos, $14 \%$ de 6 a 8 anos e $11 \%$ têm um tempo de trabalho de mais de 9 anos de serviço ao Ministério da Saúde Pública como técnico sênior na atenção primária. 
A técnica utilizada na presente investigação foi a pesquisa. Para isso, foi utilizada a ferramenta Maslach Burnout Inventory, que é uma das ferramentas mais utilizadas nos ambientes acadêmico, de saúde e de trabalho. Etc. Isso enfoca a medição do nível da Síndrome de Burnout ao qual os indivíduos em uma determinada situação estão expostos.

Essa ferramenta consiste em 22 itens ou perguntas, nos quais o sujeito deve marcar em uma escala do tipo Likert que varia de 1 a 5 . A opção de marcação $\mathrm{n}^{0} 1$ representa que o fenômeno "nunca" é uma fonte de atrito; A opção 2 representa que o fenômeno "algumas vezes por ano" é uma fonte de Burnout; a opção $\mathrm{n}^{\circ} 3$ representa que o fenômeno "algumas vezes por mês" é uma fonte de Burnout, a opção $\mathrm{n}^{\circ} 4$ representa que o fenômeno "algumas vezes por semana" é uma fonte de Burnout e a opção de marcação $n^{\circ} 5$ representa que o fenômeno “diário" é fonte de Burnout (Moreno-Jiménez, RodríguezCarvajal e Escobar, 2001).

O Inventário de Burnout de Maslach mede o Burnout através de três dimensões que são: exaustão emocional incorporada nos itens 1, 2, 3, 6, 8, 13, 14, 16,20; despersonalização nos itens 5, 10, 11, 15, 22; conquista pessoal refletida nos itens 4, 7, 9, 12, 17, 18, 19, 21 (Reynaga-Estrada, Mena, Valadez, Rodríguez e González, 2017).

A confiabilidade e validade do instrumento foram estatisticamente altas; é por isso que tem sido usado em algumas mídias locais e internacionais; É o caso de um estudo descritivo em saúde no Hospital Municipal de BolívarColômbia, em que o MBI apresentou consistência interna de 0,90, segundo a medida de Alfa de Cronbach (Bedoya, 2017). Outro caso de uso do instrumento é o Peru, em que em uma amostra composta por médicos, enfermeiros, técnicos, foi obtida validade de construto em relação ao uso da ferramenta em países como Chile, México, Argentina, Cuba e Bolívia (Vásquez-Manrique, Maruy-Saito e Verne-Martin, 2014). 
Por si só, o MBI também é uma ferramenta usada nos países europeus; É o caso do estudo Burnout na equipe de enfermagem do Hospital Ramón y Cajal em Madri-Espanha, em que a validade do conteúdo é significativa em relação à transformação do instrumento no idioma espanhol (Sánchez \& Sierra, 2014).

\section{RESULTADOS}

$\mathrm{Na}$ presente investigação, o processo de validação estatística foi realizado utilizando as medidas de dispersão. Este procedimento foi realizado para medir o nível de confiabilidade e validade da ferramenta aplicada à amostra. Portanto, o nível de consistência interna apresentado pela ferramenta foi de 0,79, de acordo com o alfa de Cronbach. Quanto à validade do instrumento, foi realizada uma análise fatorial, que apresentou escore de 0,62 segundo a medida de Kaiser-Meyer-Olkin (KMO); O teste de esfericidade de Bartlett obteve um grau de significância igual a 0,0 e sua média de comunalidades é de 0,59 .

No presente estudo, a dimensão de exaustão emocional obteve 22 pontos, o que, de acordo com a escala do teste MBI, significa que o nível de Burnout nesta seção é de nível médio. A dimensão despersonalização de 7; Ele o localiza em um nível médio e a dimensão de realização pessoal obteve uma pontuação de 36, o que o coloca em um nível médio. As três dimensões que compõem o diagnóstico da Síndrome de Burnout colocam os Técnicos da Atenção Básica em um nível médio; é por isso que essa situação pode desencadear uma possível transferência de nível, pois está muito próxima do nível alto e não do baixo. Por si só, dentro dos gatilhos de sua manifestação estão o estresse recorrente no trabalho, sobrecarga de trabalho, estilos de liderança, fadiga no trabalho, falta de apreciação, baixos níveis de autoestima, irritabilidade, fadiga abrangente, falta de autonomia, ansiedade; é por isso que esses elementos não são considerados estranhos ao desenvolvimento 
da síndrome nos técnicos de atenção básica, pois fazem parte de um fenômeno que é evidente no setor de saúde social segundo determinadas investigações (Bogarín, Valle, Suárez, \& López, 2016).

\section{CONCLUSÕES}

O nível a que os Técnicos de Atenção Básica estão expostos na amostra mencionada refere-se a um nível médio da Síndrome de Burnout, porém os escores obtidos com a ferramenta mencionam que há uma tendência de aumento em relação ao sofrimento maior. grau da síndrome. Por isso, é necessário tomar medidas precoces antes do desenvolvimento desse fenômeno para evitar sua complicação. O aspecto teórico da Síndrome de Burnout continua sendo um termo pouco conhecido, não apenas por causa de sua prevalência no idioma inglês, mas também por ignorância organizacional, não há mais estudos sobre o assunto em nosso ambiente organizacional generalizado. Os benefícios do método descritivo permitem que um fenômeno seja analisado de maneira desfragmentada usando uma ferramenta comprovada, porém estudos transversais apenas fornecem uma visão única do desenvolvimento do fenômeno, o que pode ser considerado uma limitação. A ferramenta Maslach Burnout Inventory (MBI) possui características sólidas em relação à sua estrutura; portanto, garante a medição do fenômeno Burnout, pois permite ao sujeito executá-lo; mostrar seu status em uma situação de trabalho (Losada, Curitol, Astudillo, Schneeberger \& Coronado, 2018). É importante notar que as causas da Síndrome de Burnout não são transcendentais e padrão nas organizações; No entanto, pesquisas realizadas na América Latina confirmam que entre 30\% e $40 \%$ dos profissionais de saúde sofrem com isso. (Arteaga \& Espinoza, 2014). Nos países latinoamericanos, foram realizados estudos sobre Burnout em professores e profissionais de saúde; para os quais os resultados fornecem pontuações altas 
e médias para a existência do fenômeno (Serrano-Díaz, Pocinho e AragónMendizábal, 2018); Por outro lado, em nosso país, é a primeira intervenção para medir esse fenômeno nos Técnicos da Atenção Básica. Seguindo essa ordem de idéias, deve-se notar que, em estudos sobre o fenômeno Burnout, as variáveis sociodemográficas são geralmente consideradas, mas isso depende do objetivo e do escopo da pesquisa (Vega, et al., 2017) O TAPS é um grupo de profissionais, com cenários de alto desenvolvimento no campo da Saúde, comunidade; no entanto, as contribuições desta pesquisa são de benefício comum para o avanço de novas tendências e medidas de novos fenômenos que aparecem em seu campo e nas organizações. Ciências e disciplinas como psicologia, medicina comunitária, sociologia são aquelas que também podem considerar as descobertas desses resultados de pesquisa. As soluções alternativas para o problema oscilam para um planejamento estratégico, de natureza departamental e individual. É então que a gestão do talento humano deve desempenhar um papel de liderança, para que haja programas de socialização e conscientização sobre a gestão do estresse no trabalho, análise das consequências de altos níveis de estresse e desenvolvimento da síndrome de burnout, faça pausas ativas que envolvam exercício físico, recreação; aumentar a conscientização sobre o efeito positivo de equilibrar a vida familiar versus a vida profissional; e, acima de tudo, o impacto positivo que a gestão desse fenômeno tem no bem-estar saudável e abrangente de cada técnico e trabalhador em geral (Arteaga \& Espinoza, 2014). Por fim, pesquisas sobre estresse, estresse no trabalho e Síndrome de Burnout não são encontradas apenas nas áreas da Saúde; portanto, existem também nas áreas de negócios, esportes, ensino superior e outras áreas (Rosales \& Rosales, 2014) (De Francisco, Garcés de Los Fayos e Arce, 2015) (Loaiza \& Peña, 2013). Portanto, essa pesquisa pode servir como referência para iniciar, projetar e aprofundar pesquisas futuras relacionadas aos 
fenômenos de trabalho nos centros de atendimento do nosso ambiente; como Burnout, estresse crônico no trabalho, riscos psicossociais; tudo isso, a fim de aumentar a quantidade e a qualidade dos estudos sobre esses temas.

\section{REFERÊNCIAS}

Albendín, L., Gómez, J. C.-d., Cañadas, G., San Luis, C., \& Aguayo, R. (2016). Prevalencia bayesiana y niveles de burnout en enfermería de urgencias. Una revisión sistemática. Revista Latinoamericana de Psicología, $\quad 48(2)$, 137-145. doi:http://dx.doi.org/10.1016/j.rlp.2015.05.004

Alvarez-Silva, L., Estrella-Marín, B., \& Rosas-Baldeón, M. (2018). Rol del Psicólogo Organizacional en la gestión de Talento. PODIUM, 1(33), 79-90. doi:http://dx.doi.org/10.31095/podium.201

Arteaga, D., \& Espinoza, C. (2014). Síndrome de burnout en el personal de áreas con atención directa a clientes externos en una institución educativa. Samborondón: Universidad Espíritu Santo.

Barros-Bastidas, C., \& Turpo, O. (2020). La formación en investigación y su incidencia en la producción científica del profesorado de educa- ción de una universidad pública de Ecuador. Publicaciones, 50(2), 167185. doi:10.30827/publicaciones.v50i2.13952

Barros Bastidas, C., \& Turpo Gebera, O. (2018). Factors influencing the scientific production of university professors: a systematic review . Pensamiento Americano, 11(22). https://doi.org/10.21803/pensam.v11i21-1.276

Bedoya, E. (2017). Prevalencia del síndrome de burnout en trabajadores de un hospital público colombiano. MEDISAN, 21(11), 3172-3179. 
Bedoya, E., Vega, N., Severiche, C., \& Meza, M. (2017). Síndrome de Quemado (Burnout) en Docentes Universitarios: El Caso de un Centro de Estudios del Caribe Colombiano. Formación Universitaria, 10(6), 51-58. doi:http://dx.doi.org/10.4067/S0718-50062017000600006

Bogarín, M., Valle, R., Suárez, M., \& López, M. (2016). El Síndrome de Burnout en Educación Superior. 12(13), 230-241.

Constitución de la República del Ecuador. (2008). Sección septima, Salud. Quito: Ediciones legales.

De Francisco, C., Garcés de Los Fayos, E. J., \& Arce, C. (2015). La medida del sindrome de Burnout en deportistas: una perspectiva multidimensional. Revista Iberoamericana de Psicología del Ejercicio y el Deporte, 10(2), 173-178.

Díaz, F., \& Gómez, I. (2016). La investigación sobre el síndrome de burnout en latinoamérica entre 2000 y el 2010. Psicología desde el Caribe, 33, 113-131.

Domínguez, J., Herrera, F., Villaverde, M. d., Padilla, I., Martínez, M., \& Domínguez, J. (2011). Síndrome de desgaste profesional en trabajadores de atención a la salud en el área sanitaria de Ceuta. Atención Primaria, 44(1), 30-35. doi:http//:doi:10.1016/j.aprim.2011.01.004

Domínguez-González, A., Velasco-Jiménez, M., Meneses-Ruiz, D., Valdivia-Gómez, G., \& Castro-Martínez, M. (2017). Síndrome de burnout en aspirantes a la carrera de medicina. Investigación en Educación $\quad$ Médica, $\quad 6(24), \quad 242-247$. doi:http://dx.doi.org/10.1016/j.riem.2016.11.007 
Gómez-Urquiza, J., Monsalve-Reyesb, C., San Luis-Costas, C., FernándezCastillo, R., Aguayo-Estremera, R., \& Cañadas-de la Fuente, G. (2017). Factores de riesgo y niveles de burnout en enfermeras de atención primaria: una revisión sistemática. Atención Primaria, 49(2), 77-85. doi:https://doi.org/10.1016/j.aprim.2016.05.004

Hernández Sampieri, R., Fernández, C., \& Baptista, M. (2014). Metodología de la Investigación (Vol. 6). México: McGraw-Hill.

Loaiza, E., \& Peña, A. (2013). Niveles de estrés y síndrome de Burnout en contadores públicos colombianos. Actualidad Contable Faces, $16(26), 27-44$

Losada, H., Curitol, S., Astudillo, P., Schneeberger, P., \& Coronado, F. (2018). Prevalencia de Síndrome de Burnout en un Centro de Cirugía Académico-Asistencial Público en Chile. Revista Chilena de Cirugía, $70(2), 117-126$

Ministerio de Salud Pública - MAIS. (2012). Modelo de Atención integral de Salud. Quito: Ministerio de Salud Pública.

Moreno-Jiménez, B., Rodríguez-Carvajal, R., \& Escobar, E. (2001). La evaluacion del Burnout profesional Factorialización del MBI-GS. Un análisis preliminar. Ansiedad y Estrés, 7(1), 68-79.

Munayco-Guillén, F., Cámara-Reyes, A., \& Torres-Romucho, C. (2014). Síndrome de burnout en internos de medicina: un problema urgente. Acta Médica Peruana, 31(4), 249.

Organización Internacional del Trabajo - OIT. (2016). Estrés en el trabajo: un reto colectivo. Ginebra: Publicaciones de la OIT. 
Organización Mundial de la Salud. (24 de 07 de 2018). ¿Cómo define la OMS la salud? Obtenido de ¿Cómo define la OMS la salud?: http://www.who.int/suggestions/faq/es/

Organización Panamericana de la Salud - OPS. (2008). Marco conceptual de los Objetivos de desarrollo del Milenio desde una perspectiva de los pueblos indígenas. Washington, DC 20037: Nuevo Arte.

Patlán, J. (2013). Efecto del burnout y la sobrecarga en la calidad de vida en el trabajo. Estudios Gerenciales, 29(129), 445-455.

Reynaga-Estrada, P., Mena, D., Valadez, A., Rodríguez, A., \& González, J. (2017). Síndrome de burnout en deportistas universitarios mexicanos. Revista de Psicología del Deporte, 26(2), 209-214.

Rosales, Y., \& Rosales, F. (2014). Hacia un estudio bidimensional del Síndrome de Burnout. Ciência \& Saúde Coletiva, 19(12), 4767-4775.

Ruiz, E., Gago, M., García, C., \& López, S. (2012). Recursos humanos y responsabilidad social corporativa (1a ed.). Madrid: McGraw-Hill.

Sánchez, P., \& Sierra, V. (2014). Síndrome de Burnout en el personal de enfermería en UVI. Enfermería Global, 13(1), 252-266.

Serrano-Díaz, N., Pocinho, M., \& Aragón-Mendizábal, E. (2018). Competencias emocionales y síndrome de burnout en el profesorado de Educación Infantil. Revista de Psicología y Educación, 13(1), 112. doi:http://dx.doi.org/10.23923/rpye2018.01.153

Teixeira, C., Gherardi-Donato, E. d., Pereira, S., Cardoso, L., \& Reisdorfer, E. (2016). Estrés laboral y estrategias de afrontamiento entre los profesionales de enfermería hospitalaria. Enfermería Global, 15(4), 288-298. 
Vásquez-Manrique, J., Maruy-Saito, A., \& Verne-Martin, E. (2014). Frecuencia del síndrome de Burnout y niveles de sus dimensiones en el personal de salud del servicio de emergencia del pediatría del Hospital Nacional Cayetano Heredia en el año 2014. Lima, Perú. Revista de Neuro-Psiquiatría, 77(3), 168-174.

Vega, P., González, R., Bustos, J., Rojo, L., López, M., Rosas, A., \& Hasbún, C. (2017). Relación entre apoyo en duelo y el síndrome de Burnout en profesionales y técnicos de la salud infantil. Revista chilena de Pediatría, 614-621. doi:http://doi:10.4067/S037041062017000500007

von Feigenblatt, Otto Federico, ASEAN and Human Security: Challenges and Opportunities (July 29, 2009). Ritsumeikan Center for Asia Pacific Studies Working Paper, No. 09-5, Available at SSRN: https://ssrn.com/abstract=1442476 or http://dx.doi.org/10.213 9/ssrn.1442476

von Feigenblatt, Otto Federico, Japanese Animation as a Global Product: The Lingering Traces of Nijonjinron and the Rise of Globalism and Hybridity (August 2, 2012). Journal of History \& Social Sciences, 2(2), pp. 1-14, July-December 2012, Available at SSRN: $\underline{\text { https://ssrn.com/abstract }=2195562}$

von Feigenblatt, Otto Federico, Garcia Marquez' Magical Realism as a Representation of Latin America's Socio-Political Reality: Developmental Simultaneity and Exceptionalism in Latin America as Expressed in Historiographic Metafiction (December 27, 2009). The Expression, Vol. 2, No. 1, pp. 1-6, 2009, Available at SSRN: $\underline{\text { https://ssrn.com/abstract }=1596690}$ 